\title{
The optimal path optimization choice and simulation based on the labeling algorithm
}

\author{
Di Liang ${ }^{1, a}$, Bin Wang ${ }^{1, b^{*}}$ \\ ${ }^{1}$ School of Mechanical Engineering, Shenyang University, Shenyang, Liaoning Province, China \\ ${ }^{2}$ School of Mechanical Engineering, Shenyang University, Shenyang, Liaoning Province, China \\ asydxld@163.com, b1689291798@qq.com
}

Keywords: Label algorithm, Traffic optimization, Optimal path selection

Abstract. This paper uses a university traffic path optimization choice as the research object to analysis the traffic situation. It use the labeling algorithm and the Dijkstra algorithm. The Matlab is used to programme and operate the results. It is mainly solved the problem of optimal route choices and several major roads crowded in peak time to optimize the campus traffic. It greatly saved the traveling time and solved the problem of peak crowded and traffic safety.

\section{Introduction}

In recent years, various of equal educational, on-the-job learning and correspondence teach lead to social workers flood into the campus. It brings enormous pressure to the campus traffic. One university is an important examination point of national certification and all kinds of social examinations, in which most people have as many as 27 times when certification exams. Additionally the peak flow caused by the college students' when they finish classes brings some problems:for example the campus road congestion, campus staff travel safety, etc.

University campus road traffic system is a organic integral, it has the users, traffic tools and the external environment. It is a multiple factor interaction, interconnected transportation system. It assume the function of campus internal transport links with the outside world [1]. Because life and live places are in relatively concentrated. Eating and classes times are consistent relatively. Staff and students work periodic in the school and transform the classroom in break time are flow of dense. So there will arise the peak time of individuals and traffic flow in campus which is easy to come up the traffic accident [2].Roadside advertising plays an important role, it is the amount of information and traffic signs, the complexity of the background and traffic flow density together form the logo will be the main focus of the factors [3-5].The beginning of environmental deterioration among the campus traffic to make the domestic scholars also begin to focus on the campus as the traffic problem of research .Aim at the campus traffic getting more and more complex in these years, vehicles are increasing, the campus conflict and safety issues has increased with it. Putting forward the idea of people-oriented [6].

This paper combined with a university' s actual situation. It finds out the problems from the point of the campus traffic optimization and through the analysis of traffic conditions in campus Using the labeling algorithm tool to improve traffic conditions, the improvement effect in aspects of travel time and campus order is verified and compared.

\section{Optimal Path}

Selecting a right route in the university campus will bring great convenience to campus staff travel. The choice of the optimal path can provide a reference basis for campus guide signs. In general, the shortest paths is the first choice for pedestrians and vehicles on campus, if everyone choose s the shortest path, then the road traffic is particularly large, the shortest path to the destination is not always the best route, because people finally expect that the destination is the shortest time. Because the time from starting point to the end and the distance is directly proportional. It will be longer with the increased of road traffic Use $D(i, j)$ express the distance between any two nodes, use $Q(i, j)$ to 
mark the flow between any two nodes, use $T(i, j)$ to identify the travel time between any two points, the function is followed :

$$
\left.\operatorname{Min}\{T(i, j)\}=\min \{D(i, j)\}^{*} Q(i, j)\right\}^{*} K
$$

$\mathrm{i}, \mathrm{j}-$ Any two traffic nodes. $\mathrm{K}$ is scale constants.

According to the specific condition of the university the network abstraction diagram is shown in figure 1.Respectively to establish the corresponding node distance matrix and node flow matrix ( $\infty$ for two nodes not connected directly).

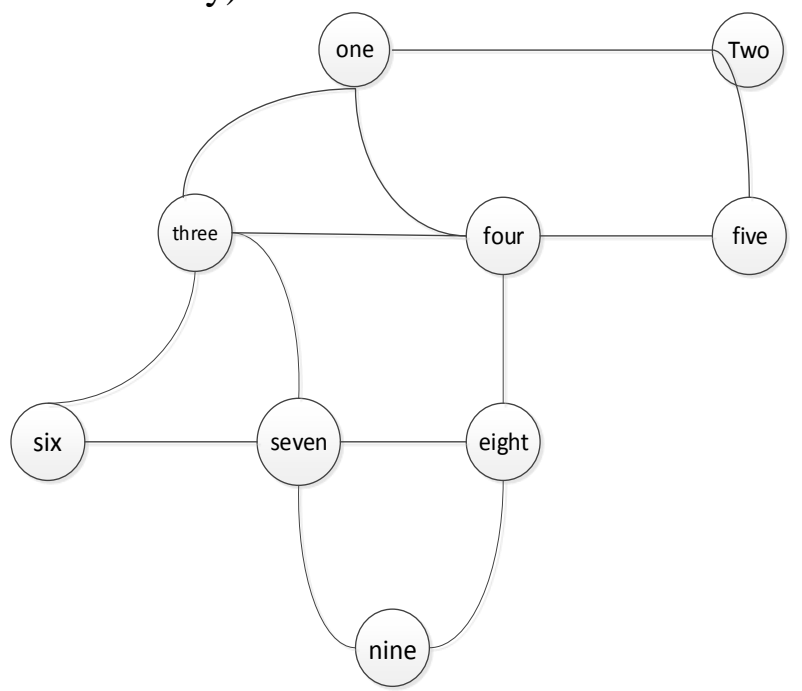

Fig. 1 Network abstract

Distance matrix :

$$
\left|\begin{array}{ccccccccc}
0 & 277 & 212 & 266 & \infty & \infty & \infty & \infty & \infty \\
277 & 0 & \infty & \infty & 156 & \infty & \infty & \infty & \infty \\
212 & \infty & 0 & 164 & \infty & 215 & 92 & \infty & \infty \\
266 & \infty & 164 & 0 & 198 & \infty & \infty & 91 & \infty \\
\infty & 156 & \infty & 198 & 0 & \infty & \infty & \infty & \infty \\
\infty & \infty & 215 & \infty & \infty & 0 & 135 & \infty & \infty \\
\infty & \infty & 92 & \infty & \infty & 135 & 0 & 211 & 114 \\
\infty & \infty & \infty & 91 & \infty & \infty & 211 & 0 & 141 \\
\infty & \infty & \infty & \infty & \infty & \infty & 114 & 141 & 0
\end{array}\right|
$$

About the optimal problem of two fixed points can use Dijkstra algorithm. The basic idea is according to the distance $U_{0}$ from the far to close. In sequence to get $U_{0}$ to $G$ for the each vertex of the short circuit and the distance. Until $V_{0}$ (or all the verticals of $\mathrm{G}$ ), algorithm ends.

(1) $l\left(u_{0}\right)=0, v \neq u_{0}, l(v)=\infty, s_{0}=\left\{u_{0}\right\}, i=0$.

(2) $v \in s_{i}^{\prime} \quad\left(s_{i}^{\prime}=V / s_{i}\right)$,

use $\min _{u \in s_{i}}\{l(v), l(u)+w(u v)\}$ instead of $l(v)$ to calculate $\min _{u \in s_{i}}\{l(v)\}$, the minimum value to $u_{i 11}, s_{i 11}=s_{i} \bigcup\left\{u_{i 11}\right\}$.

(3) $i>|V|-1$, stop;

(4) $i<|V|-1$, use $i_{11}$ instead of $i$, turn to (2).

At the end of the algorithm, the distance from $u_{0}$ to $v$ each vertex of the final grade $l(v)$ is given. Before $v$ entering $S_{i}$ the label $l(v)$ called T label and $v$ entering $s_{i}$ the label is $l(v)$ called P label. Algorithms are modify the point T label constantly, until gains $\mathrm{P}$ label. If in the process of algorithm run, obtaining the $\mathrm{P}$ label at each vertex origin edge indicated on the diagram. When the end of the algorithm, $u_{0}$ to the every points of the short route also marked on the diagram. 
Using $a_{n \times n}$ matrix ( $\mathrm{n}$ is the number of verticals) to store the right of each side of the adjacency matrix, the row vector $\mathrm{pb}$, index , index, $\mathrm{d}$ is respectively used to store $\mathrm{P}$ label information, label vertex sequence, label index and the value of the shortest path. The component:

$$
p b(i)= \begin{cases}1 & \text { When the } \mathrm{i} \text { vertex has been marked } \\ 0 & \text { When the i vertex is marked }\end{cases}
$$

Storage of index $_{2}(i)$ starting point to the $i$ point in the shortest path first vertex before a serial number; $d(i)$ deposit from starting point to the shortest path of the value of the i. At the entrance of (1)node to other nodes of the shortest path in Matlab programs and run results. In the same way the minimum flow problem between two nodes can also be converted into Dijkstra algorithm for the optimal problem. Using the Matlab program operation and operation result.

When the flow is smaller, mainly considering the shortest distance. The result is shown in figure 2.

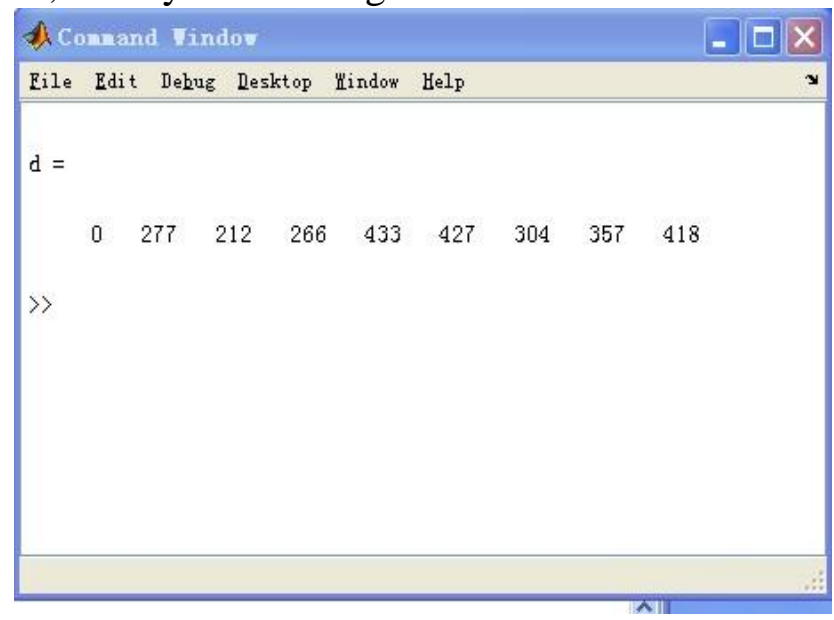

Fig. 2 Result display window

There are two ways under the path selection which is basically the same. Only the node (1) to node (5), node (1) to node (9) under the condition of two different choices of path are not the same but the rest are consistent. In terms of the school's specific situation. The basic flow to choose two paths as the real distance between them are almost the same.

\section{Campus Traffic Optimization}

Aiming at the entrance of the imperfection of the gate is narrow and guidance from the following two aspects:

(1) Shunt. Vehicles enter the campus mainly come from two directions around the school gate.

Two directions of all vehicles entering school by west gate is the main reason for the large flow. So it needs to shunt of two directions of vehicles. Vehicles from the left enter the campus through the side of sports venues, and vehicles from the right side of the vehicle enter the west campus gate.

(2) The reasonable guid ance. Mainly includes:

1) The overall plans of the campus;2) Functional zoning map; 3) Direct primary identification; 4) Auxiliary identifier

All vehicles enter into the west gate campus before improvement, the traffic flow in a half hour is 257 on workdays and 771 on weekends ; After improvement the left of the vehicle enter the campus by the sports venues, thus it greatly reduces the time that vehicles enter the campus. Since the vehicles enter campus from two gates at the same time, the consuming time will be overlapped. So the actual time that the vehicles enter the campus fully satisfies the function $t=\max \left(t_{1}, t_{2}, t_{3} \ldots . . t_{i}\right)$, $t_{i}=$ rate $\times$ flow velocity. Before improvement the total time is 1800 seconds on workdays, after improvement, $t=147 \times(1800 / 257) \mathrm{s}=882.57 \mathrm{~s}$, it saves time $t=1800 \mathrm{~s}-882.57 \mathrm{~s}=917.43 \mathrm{~s}$, the total time is 1800 seconds on weekends, after improvement, $t=471 \times(1800 / 771-1)=628.61 \mathrm{~s}$, it saves time $t=1800 \mathrm{~s}-628.61 \mathrm{~s}=1171.39 \mathrm{~s}$. The dealership time comparison between before and after improvement is shown in figure 3 . 


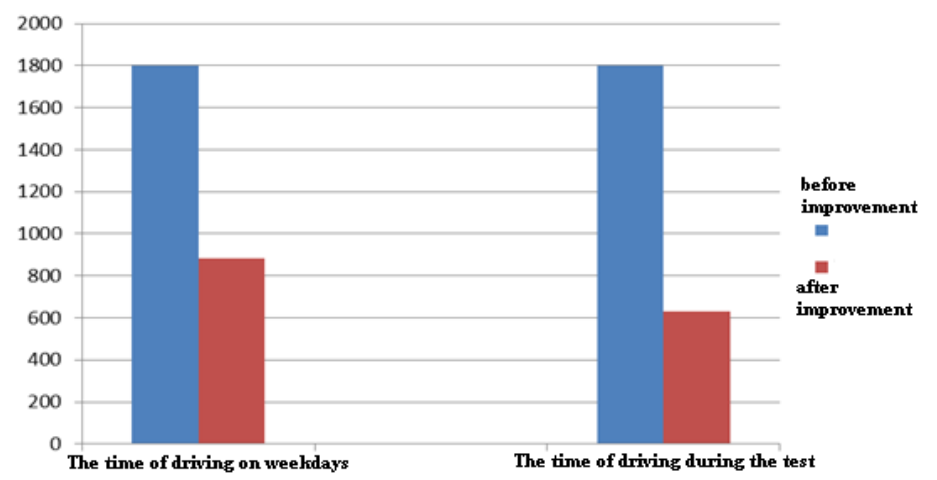

Fig 3. Driving time contrast

The coexistence of people and vehicles not only hinder the campus motorized traffic, but also relate to the security issues. So it should be focused on.

(1)Separating pedestrians and vehicles

Campus carried out slow zone and mix zone. The slow zone mainly includes the living area, teaching building, library and central green square for no motor vehicle areas (special vehicles can enter in prescribed time). Adopt the method of physical isolation to let this place become no cars safety area. Mix zone mainly includes the office building that has the function of parking, teaching building and the south green belts.

The transportation vehicles and special vehicles can enter the campus into chronic area within the given time. But it must avoid the pedestrians according to the speed limit signs.

(2) Identify guidance

Separating pedestrians and vehicles is a good way to improve the campus traffic. The separation of people and motor vehicles reduces the positive contact with the car to improve the safety of the pedestrians. But for two individuals walking in campus, it is necessary to learn campus traffic routes. Comprehensive considerate the various factors, placing a variety of campus proper guidance.

\section{Summary}

Through the choice of the optimal path in campus and the analysis of the main road traffic investigation, this paper use the label algorithm and Matlab simulation technology to improve traffic conditions, it can save $917.43 \mathrm{~s}$ on workdays and save $1171.39 \mathrm{~s}$ on weekends. The traffic safety situation of teachers and students in campus has improved significantly.

\section{References}

[1] Q. Zhang, L.H. Duan, University campus traffic safety management quality fuzzy comprehensive evaluation system research, Journal of soft science. 2 (2011) 141-144.

[2] L.P. Ding, The campus traffic status and management countermeasures, Journal of changzhou institute of technology. 1 (2011) 54-58 .

[3] P.K. Hughes, B.L. Cole, What attracts attention when driving, Ergonomics. 29(1986) 377-391.

[4] V.C. Liu, A simulated study on the effects of information volume on traffic signs, viewings catagies and sign military upon drivel's visual search performanc, Industrial Ergonomics 35 (2005) 115-147.

[5] W.A. Macdonald, E. R. Hoffinann, Drivers awareness of traffic sign information, Ergonomics. 34 (1991) 586-612.

[6] B.J. Luo, S.Q. Li, The campus road traffic system layout optimization study, China water transport. 10 ( 2009) 13-17. 